\title{
Alzheimer's disease neuropathological change and loss of matrix/neuropil in patients with idiopathic Normal Pressure Hydrocephalus, a model of Alzheimer's disease
}

\author{
Sylwia Libard ${ }^{1,2}$ and Irina Alafuzoff ${ }^{1,2^{*}}$
}

\begin{abstract}
Here, we assessed unique brain tissue samples, obtained from living subjects with idiopathic Normal Pressure Hydrocephalus (iNPH). Our cohort of 95 subjects with age ranging from 75 to 79 years, displayed a high prevalence of $\beta$-amyloid (A $\beta$ ) and hyperphosphorylated $\tau$ (HPT) pathology (63 and 61\%, respectively) in a frontal cortex biopsy obtained during shunt operation. These lesions, i.e., Alzheimer's Disease Neuropathologic Change (ADNC), increased within 5 years and were more frequent in females. The extent of HPt pathology was sparse, primarily seen as neurites and stained dots. Noteworthy, concomitant pathology was seen in $49 \%$ of the whole cohort, indicating a severity of ADNC corresponding to a low/intermediate level following the current recommendations. This observation is predictable as based on previous publications a substantial number of subjects with iNPH over time develop AD. Thus, iNPH can be considered as a model of AD. We noted a surprisingly remarkable neuronal preservation assessing Neuronal Nuclei (NeuN) in parallel with a substantial depletion of matrix/neuropil. This finding is intriguing as it suggests that loss of matrix/neuropil might be one of the first lesion of ADNC but also a hallmark lesion of iNPH. The latter observation is in line with the enlarged ventricles, a cardinal feature of iNPH. Furthermore, a positive correlation was observed between the extent of $A \beta$ and NeuN but only in females indicating a neuronal preservation even when $A \beta$ pathology is present. The assessment of a surgical biopsy as described here is certainly informative and thus it is surprising that a neuropathologic assessment in the setting of iNPH, while inserting a shunt, is seldom performed. Here, we observed ADNC and surprisingly remarkable neuronal preservation in a substantial number of iNPH subjects. Thus, these subjects allow us to observe the natural course of the disease and give us an opportunity for intervention at the earliest stages of $A D$, prior to severe neuronal damage.
\end{abstract}

Keywords: Alzheimer's disease Neuropathologic change, Idiopathic Normal pressure hydrocephalus, Neuronal loss, Matrix/neuropil, Immunohistochemistry

\footnotetext{
* Correspondence: irina.alafuzoff@igp.uu.se

'Department of Immunology, Genetics and Pathology, Uppsala University,

Rudbeck Laboratory, Dag Hammarskjölds väg 20, 751 85, Uppsala, Sweden

${ }^{2}$ Department of Pathology, Uppsala University Hospital, Uppsala, Sweden
}

(c) The Author(s). 2019 Open Access This article is distributed under the terms of the Creative Commons Attribution 4.0 International License (http://creativecommons.org/licenses/by/4.0/), which permits unrestricted use, distribution, and reproduction in any medium, provided you give appropriate credit to the original author(s) and the source, provide a link to the Creative Commons license, and indicate if changes were made. The Creative Commons Public Domain Dedication waiver (http://creativecommons.org/publicdomain/zero/1.0/) applies to the data made available in this article, unless otherwise stated. 


\section{Introduction}

The idiopathic normal pressure hydrocephalus (iNPH) is a neurological condition affecting elderly patients, with symptoms including gait disturbance, cognitive impairment (CI), and urinary incontinence [42]. The diagnosis is based on the existence of the above given symptom triad, detection of enlarged ventricles on imaging, and pathological cerebrospinal fluid (CSF) dynamic tests [42]. Up to date, no characteristic neuropathologic lesion or post mortem (PM) applicable neuropathological consensus criteria are available for iNPH.

Previous publications have shown that a substantial number of subjects with iNPH display, in a minimal cortical biopsy obtained during ventriculoperitoneal shunt (VPS) insertion, hallmark lesions of Alzheimer's Disease $(\mathrm{AD})$. The $\mathrm{AD}$ neuropathologic changes (ADNC) include $\beta$-amyloid $(\mathrm{A} \beta)$ and hyperphosphorylated $\tau(\mathrm{HP \tau})$ aggregates, primarily seen in the gray mater $[3,5-7,13,23$, $25,31,35,40]$. Noteworthy, a substantial number of subjects with iNPH develop clinical dementia over time, particularly dementia of the AD type [20]. In line with the above, concomitant ADNC have been seen in PM neuropathological examination in a subset of patients with clinical diagnosis of iNPH $[11,22,25]$.

The treatment of choice of iNPH is insertion of a VPS that can, in some cases, reverse the symptoms by normalizing the CSF flow in the cerebral cavities [42]. While shunting, a catheter is inserted through the brain parenchyma, usually the right frontal lobe, into the lateral ventricle and the opposite tip of the catheter is directed towards the peritoneal space. There is a shunt valve between the catheter ends, opening when the CSF pressure is excessively high, aiming to reach a physiologic equilibrium and normalize the CSF flow within the brain [42]. Thus the VPS is inserted to continuously alleviate eventual increase in the CSF pressure. Interestingly, the presence of ADNC in the biopsy has been associated with a lack of VPS response and with worse prognosis after the VPS surgery $[1,5,20,31]$.

There are a few centers in the world that have obtained a cortical biopsy during the VPS insertion; similarly, there are a few reports on PM neuropathologic finding in subjects with clinical diagnosis of iNPH $[5,11$, $13,22,23,25,31,35]$.

$\mathrm{AD}$ is the most common neurodegenerative disease causing dementia [4]. The cardinal symptoms are CI and/or memory loss, considered to be caused by a progressive synaptic and neuronal damage [28, 33, 37, 38]. Several reports indicate that the synaptic loss and neuronal damage are associated with progressive accumulation of $\mathrm{A} \beta$ and HPt in the brain tissue $[18,26,33,37$, 38]. Noteworthy, some studies describe cell loss in the early stages of $\mathrm{AD}$ [16], whereas others state that neurons can survive and function even displaying $\mathrm{HP \tau}$, also at a late stage of the disease [10]. However, it should be noted that stepwise progression of ADNC is observed in a substantial number of unimpaired subjects prior to the clinical AD syndrome but also in individuals that never progress to dementia $[8,9,14]$. Thus, the assessment of synaptic loss and neuronal loss in association with ADNC is more complex than anticipated.

Most of the studies addressing synaptic loss and neuronal loss in AD are performed on PM brain tissue, animal models, or cell lines. Regarding iNPH, there are no animal models or cell lines; moreover, the human brain tissue to be assessed is scanty. Thus, to our knowledge, these alterations have not been addressed.

The purpose of this study was to assess ADNC and neuronal loss, applying sensitive immunohistochemical (IHC) technique in surgical biopsies from frontal cortex of patients with clinical diagnosis of iNPH. The benefit of this approach is that we are investigating human tissue, i.e., a surgical sample, which is well preserved, lacking all changes that are related to PM events, including agonal state, PM delay, and long fixation time. The pitfall is the minimal size of the biopsy, but this issue was considered negligible as the objective was to assess defined alterations within a defined area.

\section{Material and methods Ethical statement}

The study was approved by the regional Ethical Committee of Uppsala, Sweden \#2013/176, updated 2016. All the subjects included have given their informed consent for the use of their tissue samples for clinical and scientific purposes.

\section{Study subjects}

The study was carried out on brain biopsies obtained for diagnostics, from patients diagnosed with iNPH and operated on at the Neurosurgery Department at the Uppsala University Hospital (UUH), Sweden, during 2010-2016. In total, brain samples from 364 subjects were identified at the Pathology Department, UUH. In addition, the subject had to be within the age range of 75 to 79 years, as the age is known to significantly influence all the alterations to be assessed [14, 32]. In total, 107 subjects were identified. Seven of these subjects displayed only white matter in their biopsies and were thus excluded. An additional five individuals were excluded due to the loss of gray matter during the processing of the tissue. Thus, brain tissue samples from 95 subjects remained for the final analysis. In addition, a set of samples based on the outcome in one of the neuronal markers were selected to be IHC stained with antibody to synaptophysin (SYP). 


\section{Brain samples}

The diagnostic brain biopsies were obtained during the VPS operation, as previously described [13, 23, 42]. The samples were taken from the right frontal lobe, more exactly, within an area of the superior- and medial- right frontal gyri. The biopsies were then fixed in $10 \%$ neutral buffered formalin ( $4 \%$ formaldehyde) for $24 \mathrm{~h}$ at room temperature and processed into paraffin (Histowax from Histolab) blocks. The blocks were sectioned on the microtome from Thermo Scientific (Microm HM 355S) into $4 \mu \mathrm{m}$ thick sections, which were placed on Super Frost slides for standard Hematoxylin-Eosin and Super Frost Plus slides for IHC stainings.

\section{Immunohistochemistry}

The IHC stainings were carried out automatically or manually. The automated stainings were performed on the Dako Autostainer Plus (DakoCytomation, Glostrup, Denmark) using the Dako EnVision Flex detection system (DakoCytomation, Glostrup, Denmark), according to the manufacturer's instructions. The Bright Vision detection system (IL Immunologic, Duiven, The Netherlands), with a Romulin AEC for antigen detection (BioCare Medical, Pacheco, CA, U.S.A.), was used for the manual stainings. The antibodies $(\mathrm{Ab})$ used and the pretreatment strategies applied are listed in Table 1.

\section{The analysis of the samples}

Primarily, all biopsies were assessed using light microscopy (Olympus BX45) at magnification $\times 20$ to $\times 400$. The samples were then scanned into digital slides, saved as ScanScope Virtual Slide (svs) format, with Aperio AT2 (Leica Biosystems) scanning system in 20x magnification. For the morphometric image analyses, the Aperio ImageScope software (Leica Biosystems) was used, applying the positive pixel count algorithm (version 9.1) for quantification of the immunoreactivity (IR). All parameters were preset in the software, except "The Intensity Threshold (Upper Limit) of WEAK positive pixels," which was increased from 220 to 255 .

A color code was used to visualize the staining intensity, where negative pixels were blue, the week staining was visualized in yellow, moderate in orange, and the strong staining intensity was brown. The algorithm was applied on the gray matter area in each biopsy. The molecular layer of the gray matter and vessels with cerebral amyloid angiopathy (CAA) were not included in the analysis when present in the sample. The IR positive pixels were calculated in the assessed gray matter area and transformed to stained area in $\mathrm{mm}^{2}$. The number of IR pixels, i.e., pixels of given intensity, counted in the analysis was dependent of the staining quality and the compartment of the structure that expressed the protein of interest. The sum of all the positive pixels, i.e., weak, moderate, and strong, was assessed for A $\beta$. In HPт - and embryonic lethal abnormal visual system proteins 3 and 4 human homolog $\mathrm{HuC} / \mathrm{HuD}(\mathrm{HuC} / \mathrm{HuD})$ stainings, the moderate- and strong positive pixels were included. Only the strong positive pixels were counted for the Neuronal Nuclei (NeuN) and SYP staining. The final quantification of the IR in the tissue is given as a stained area fraction (SAF), a ratio between the stained IR area per total area analyzed in the biopsy $\times 100$.

\section{Statistical analysis}

IBM SPSS statistics (version 25) was used for the statistical analyses. Here, we used non-parametric tests such as Mann Whitney $\mathrm{U}$ test (MWU) and Kruskal Wallis test (KWT) to assess the differences between the genders and different age groups. Spearman's rho two tail test was used to define the correlation between the studied variables.

\section{Results}

Ninety-five individuals, 45 females and 50 males, between the ages of 75 to 79 were included in this study. The mean age \pm standard error of means $(\mathrm{m} \pm \mathrm{SE})$, at biopsy for the whole cohort was $77.03 \pm 0.14$ years, for females $76.96 \pm 0.21$, and for males $77.10 \pm 0.2$ years. No significant age difference was observed between the genders (MWU). The total area analyzed in the frontal cortex biopsies, represented by the gray matter, varied from 0.16 to $38.00 \mathrm{~mm}^{2}\left(7.69 \pm 0.33 \mathrm{~mm}^{2}\right)$.

Microscopic evaluation displayed no inflammatory processes, tumors, or extensive bleeding, alterations that might affect the interpretation of the pathology to be

Table 1 Immunohistochemical stains

\begin{tabular}{|c|c|c|c|c|}
\hline Antibody & Clone & Company/Code & Dilution & Pretreatment \\
\hline Amyloid $\beta(A \beta)$ & $6 \mathrm{~F} / 3 \mathrm{D}$ & Dako-Agilent/M0872 & $1: 50$ & $98-100 \%$ FA \\
\hline $\begin{array}{l}\text { Embryonic lethal abnormal visual system proteins (nELAV) } 3 \\
\text { and } 4 \text { human homolog HuC/HuD (HuC/HuD) }\end{array}$ & $16 \mathrm{~A} 11$ & ThermoFisher Scientific/A-21271 & $1: 2000$ & $\mathrm{ac}, \mathrm{CB}$ \\
\hline NEUronal Nuclei (NeuN) & A60 & Milipore/MAB377 & $1: 2000$ & $\mathrm{CB}$ \\
\hline Hyperphosphorylated (Ser202/Thr205) T (TAU8) & PHF-TAU-AT8 & Fisher Sientific-Invitrogen/MN1020 & $1: 1000$ & \\
\hline Synaptophysin (SYP) & SY38 & Dako-Cytomation/M0776 & $1: 50$ & $\mathrm{CB}$ \\
\hline
\end{tabular}

Dako Autostainer Plus (Dako Cytomation) was used for A $\beta$, Tau8 and SYP, while the HuC/HuD and NeuN were carried out manually, incubation at room temperature for $1 \mathrm{~h}$. Autoclave (ac), formic acid (FA), citrate buffer pH 6.0 (CB) 
assessed within the tissue. CAA was noted in four out of the 95 cases (4\%), not included in the SAF values below. The presence of $A D N P$, i.e., $A \beta$ and $H P \tau$, was verified by light microscopy in $\times 20-\times 400$ magnification. Light microscopic assessment of the staining outcome while applying the neuronal markers $\mathrm{NeuN}$ and $\mathrm{HuC} / \mathrm{HuD}$ confirmed the neuronal compartmentalization and SYP localization into neuropil.

IHC/A $\beta$ was seen in 60 out of the $95(63 \%)$ biopsies: 32 of $45(71 \%)$ females and 28 of $50(56 \%)$ males. The extent of the IHC/A $\beta$ varied from a single aggregate to multiple diffuse and compact aggregates within the tissue, with the SAF ranging from $\sim 1$ to $27 \%$ (Fig. 1a-d). The SAF/A $\beta$ was significantly influenced by age, but the significant association was not observed in females (Table 2). IHC/HPt was found in 58 (61\%) biopsies: 31of $45(69 \%)$ females and 27 of $50(54 \%)$ males. HPt pathology increased from a single granule or thread to multiple tangles in the neurons and/or abundant neurites within the neuropil, with the SAF ranging from 0.02 to $19 \%$ (Fig. 1, e-h). The SAF of HPt was less than $1 \%$ in as many as 47 of the $58(81 \%)$ samples. The SAF/HPt was significantly influenced by age but only in the whole cohort (Table 2). Concomitant IHC/A $\beta$ - and IHC/HPtpathology was observed in 47 out of 95 (49\%) subjects in our cohort; moreover, the SAF of these pathologies showed a significant correlation, for both men and women (Table 2).

A significant increase of NeuN was observed with increasing age. A significant correlation was observed between the two neuronal markers $\mathrm{NeuN}$ and $\mathrm{HuC} / \mathrm{HuD}$ (Table 2). A positive correlation $(p<0.06)$ was observed between $A \beta$ and NeuN, but only in females (Fig. 2).

When comparing the age groups, the SAF/NeuN was significantly higher in the oldest when compared with
Table 2 Spearman's rho correlations and significance ${ }^{p}$. Correlation is significant at the 0.01 level (2-tailed) is given in bold

\begin{tabular}{|c|c|c|c|}
\hline & All & Female & Male \\
\hline Number & 96 & 45 & 50 \\
\hline Age/A $\beta$ & $0.19^{0.07}$ & & $0.26^{0.07}$ \\
\hline Age/HPt & $0.18^{0.08}$ & & \\
\hline Age/NeuN & $0.49^{0.000}$ & $0.42^{0.004}$ & $0.51^{0.000}$ \\
\hline$A \beta / H P T$ & $0.58^{0.000}$ & $0.64^{0.000}$ & $0.47^{0.001}$ \\
\hline A $\beta /$ NeuN & & $0.28^{0.06}$ & \\
\hline NeuN/HuC/HuD & $0.27^{0.010}$ & & $0.26^{0.06}$ \\
\hline
\end{tabular}

$A \beta$ Amyloid $\beta, H P \tau$ Hyperphosphorylatedt, NeuN NEUronal Nuclei, HuC/HuD Embryonic lethal abnormal visual system proteins (nELAV) 3 and 4 human homolog $\mathrm{HuC} / \mathrm{HuD}$

the youngest subjects (KWT $p=0.000$ ). Contrary to the above, the age did not influence the SAF of $\mathrm{HuC} / \mathrm{HuD}$ (MWU/KWT).

The $\mathrm{m} \pm \mathrm{SE}$ of SAF of the selected four cases for the synaptic marker SYP was $95.31 \pm 0.36 \%$. The two samples with the lower values of the SAF/NeuN ( 5\%) displayed a SAF/SYP value (90 and $91 \%$ ) that was slightly higher than the SAF/SYP value (86 and $89 \%$ ) observed in the two samples with a relatively high value of SAF/ NeuN ( 10\%), as shown in Fig. 3.

The SAF of assessed proteins, in the whole cohort and in the genders, is presented in Table 3. There was a significant difference in the SAF/HPt between the genders, where females displayed higher values of SAF/HPt. The females also had higher SAF/A $\beta$ values (borderline significant). Furthermore, a borderline difference was observed for $\mathrm{SAF} / \mathrm{Huc} / \mathrm{HuD}$, whereas no difference was seen between the genders for the SAF/ NeuN.
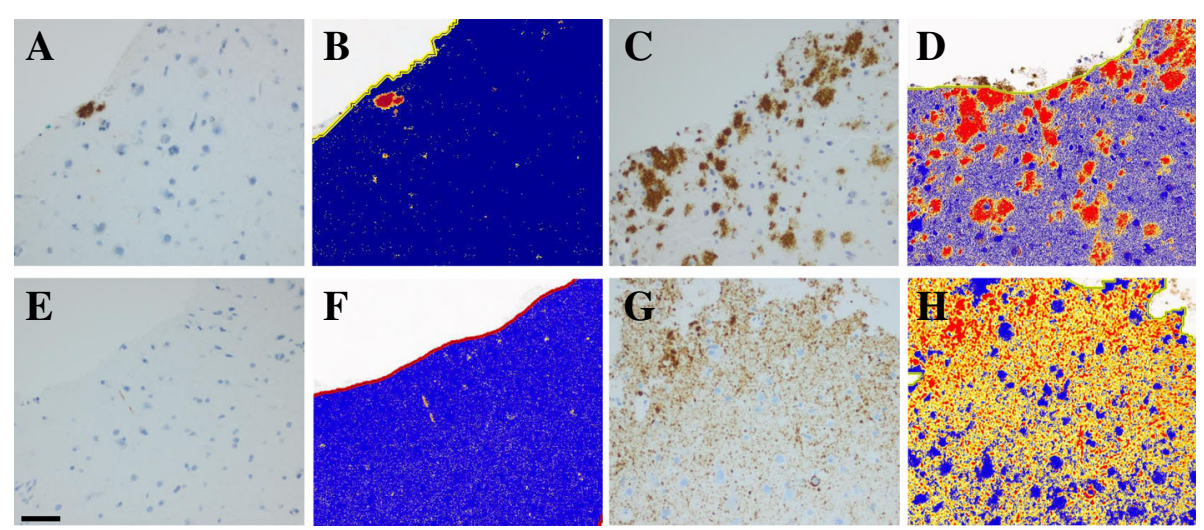

Fig. 1 Photos of immunohistochemically $(\mathrm{IHC})$ stained sections of brain biopsies from the frontal cortex. In a-d, the IHC outcome when applying antibodies directed to the $\beta$-amyloid (A $\beta$ ) and in $\mathrm{E}-\mathrm{H}$ applying hyperphosphorylated $\tau$ protein (HPT). In $\mathbf{b}$, $\mathbf{d}$, $\mathbf{f}$, and $\mathbf{h}$, the results of positive pixel count analysis, where negative pixels are blue, weak stained positive pixels are yellow, moderately stained pixels are orange, and strong positive pixels are brown. In $\mathbf{a}-\mathbf{b}, \mathrm{A} \beta$ extent in a case with stained area fraction (SAF) of 1\%; in C-D A 3 extent SAF 27\%; E-F HPt extent SAF 0.05\% and in G-H HPt extent SAF 19\%. Bar $50 \mu \mathrm{m}$ 


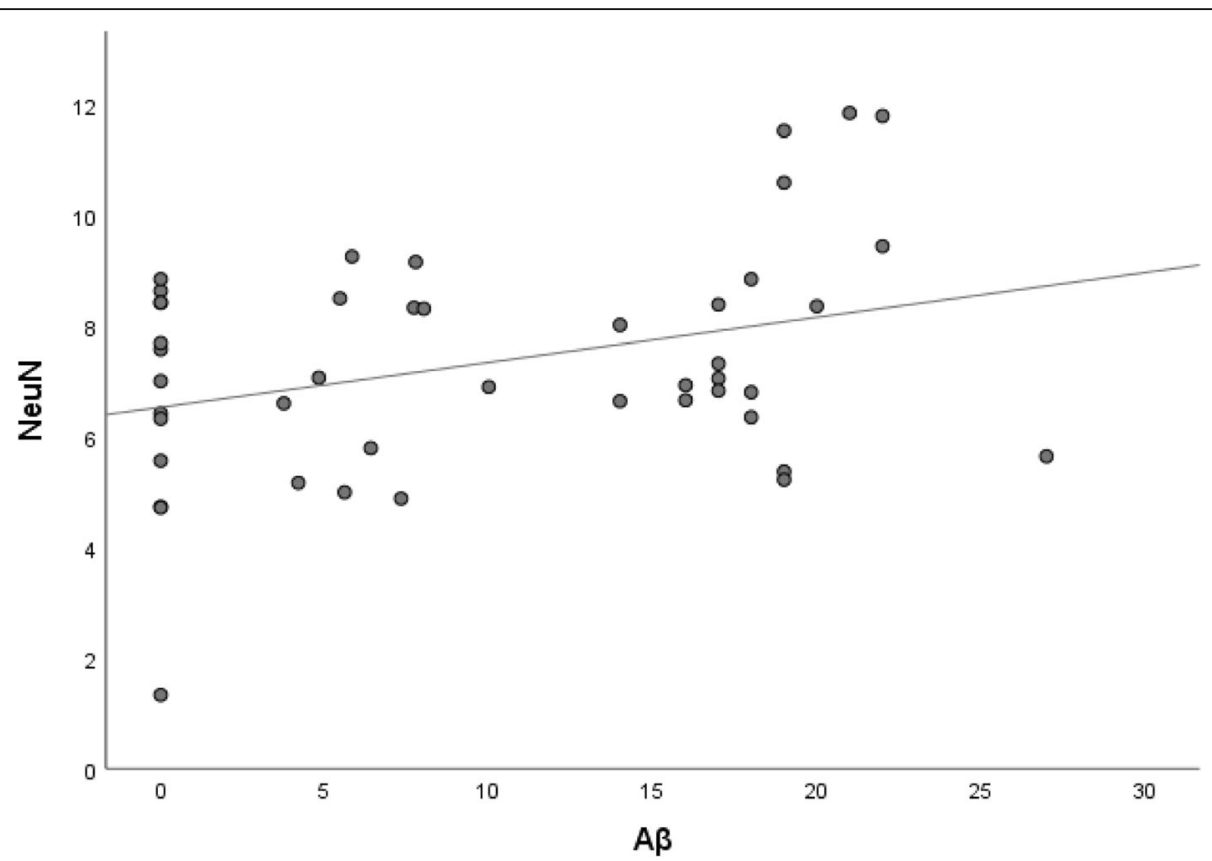

Fig. 2 Dot plot to illustrate the correlation between stained area fraction (SAF) of Neuronal Nuclei (NeuN) and SAF of $\beta$-amyloid (A $\beta$ ) in 45 females with idiopathic Normal Pressure Hydrocephalus. Spearman's rho correlation coefficient $=0.28, p=0.06$
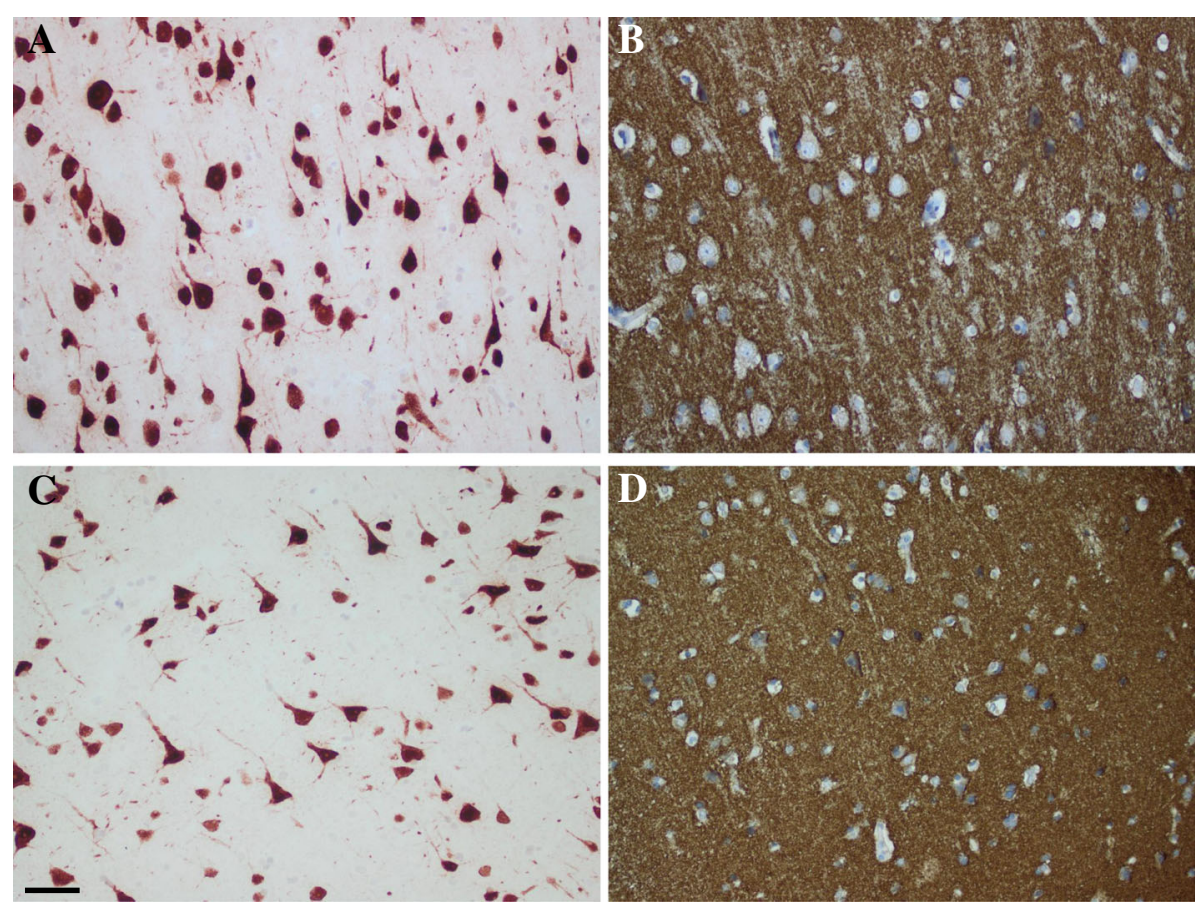

Fig. 3 Photos of immunohistochemically $(\mathrm{IHC})$ stained sections of brain biopsy from the frontal cortex, applying antibody directed to the Neuronal Nuclei (NeuN) or synaptophysin (SYP). In a and c, NeuN stained area fraction (SAF), in A 10\% and C 5\%. Note the significant difference in neurons to be seen in the same area. In $\mathbf{b}$ and c, SYP SAF in B $86 \%$ and in D 90\%. Note in $\mathbf{b}$, the loss of granular staining of SYP when comparing with $\mathbf{d}$. Bar $50 \mu \mathrm{m}$ 
Table 3 Stained area fraction (SAF) in percent, for the antibodies used, when comparing females and males. Significance level is 0.05 is given in bold

\begin{tabular}{llllll}
\hline & Value & All & Females & Males & Statistics/MWU \\
\hline Number & & 95 & 45 & 50 & \\
A & $m \pm S E$ & $8.30 \pm 0.84$ & $9.93 \pm 1.25$ & $6.83 \pm 1.11$ & $p=0.060$ \\
HPt & $m \pm S E$ & $1.04 \pm 0.32$ & $1.51 \pm 0.57$ & $0.62 \pm 0.33$ & $\boldsymbol{p}=\mathbf{0 . 0 2 2}$ \\
NeuN & $m \pm$ SE & $7.50 \pm 0.23$ & $7.34 \pm 0.30$ & $7.68 \pm 0.35$ & ns \\
HuC/HuD & $m \pm$ SE & $8.74 \pm 0.29$ & $8.38 \pm 0.42$ & $9.08 \pm 0.40$ & $p=0.060$
\end{tabular}

A $\beta$ Amyloid $\beta, H P \tau$ Hyperphosphorylated, NeuN NEUronal Nuclei, HuC/HuD Embryonic lethal abnormal visual system proteins (nELAV) 3 and 4 human homolog HuC/HuD, Syp, Synaptophysin, MWU Mann Whitney U test, the significance level is at $0.05 ; \mathrm{m} \pm \mathrm{SE}$, mean \pm standard error of mean

\section{Discussion}

A substantial number of cortical biopsies studied here, obtained from 95 subjects with iNPH, exhibited ADNC, with $A \beta$ in 63 and HPt in 61\%. The frequency of subjects with $A \beta$ is in line with previous reports whereas the percent of subjects with HPt is significantly higher $[5,13,23,25,31$, 35]. One explanation to these discrepant results is the age of the cohort investigated. We chose to include subjects within the age range of 75-79 years, compared to other studies that included subjects within an age range from 28 up to 87 years [23]. We excluded the oldest to be confident that Age Related Tau AstroGliopathy (ARTAG) pathology was not present [21].

Another factor that might have influenced the finding of a high SAF/HPt in our cohort is the methodology. Visualization of HPt applying IHC and automatic platform is currently relatively secure, whereas the assessment of IR might vary. Here we used morphometric method and assessed all pathology disregarding the size of the alteration, i.e., also tiny granules were included. The SAF/HPt in our study varied from 0.02 (only few grains) to a SAF value of $19 \%$. A low value of SAF (0.05) is pictured in Fig. 1e-f, visualizing both the sparse extent and focal distribution of IR that might be disregarded without the use of imaging technique. To obtain a result in line with previous reports (10\% of subjects with HPt pathology), all subjects with sparse HPт/SAF (0.02$1.5 \%)$ should have been excluded in our cohort [23]. In summary, three aspects that have influenced the outcome are the age of the cohort, method used to visualize the lesion and assessment strategy implemented.

Our observation of a relatively high prevalence of $\mathrm{A} \beta$ - and HPt- pathology (63 and 61\%) is in line with the biology of ADNC, known for increases with chronological age $[9,14]$. Noteworthy, even in a relatively narrow age span of 5 years, from 75 to 79 years, the extent of both HPт and A $\beta$ correlated with the age at a borderline level.

In line with what has been reported in PM studies, a significant correlation was observed between the extent of hallmark lesions of $\mathrm{AD}, \mathrm{A} \beta$, and HPt. The two protein alterations of ADNC progress, following distinctive neuroanatomical regions; $A \beta$, starting in the cerebral cortex, progresses through the limbic regions of the brain toward the cerebellum, whereas HPt is seen initially in the subcortical areas, following in the limbic structures and finally in the neocortex $[3,6,7,40]$. Thus, when both these alterations, i.e., ADNC, are observed in the biopsy obtained from the cerebral cortex, the finding suggests a stage of ADNC pathology corresponding to at least low/intermediate level, following the current staging strategy $[17,27]$. These observations of a substantial number $(\sim 60 \%)$ of iNPH displaying low/intermediate level of ADNC is in line with a previous report indicating that $50 \%$ of subjects with iNPH develop dementia over time, presumably of the AD type [20].

Surprisingly, when assessing the neuronal marker NeuN, we noted an increase in the SAF with age, i.e., we observed a higher number of neurons within a defined area, i.e., more neurons in relation to the matrix/neuropil. This finding suggests that in our cohort of iNPH subjects, a significant alteration not previously reported is depletion of the matrix/neuropil. One explanation for this outcome could be consolidation of neuropil due to an eventual degenerative process, including loss of neuronal- and glial processes or depletion of extracellular matrix proteins. Both a loss of neuronal processes and a depletion of matrix proteins have been previously reported to be observed in subjects with $\mathrm{AD}$ but not in subjects with iNPH [36-38]. Thus, it is not clear whether this observation, i.e., depletion of the matrix/ neuropil is due to the ADNC alterations, seen in a high number of our cohort, or whether it is an alteration related to iNPH. Interestingly the NeuN correlated with $\mathrm{A} \beta$ at a borderline level but only in females, and this finding might be due to a more severe depletion of neuropil, i.e., neuronal processes and matrix proteins in females parallel with the increase of $A \beta$ (Fig. 2).

Synaptic density, a measure of the depletion of the neuropil, has been studied for many decades and is reported to occur in normal aging and is accelerated in different neurodegenerative diseases, particularly in $\mathrm{AD}$ $[26,37,38]$. Synaptic damage is seen early in the disease process; furthermore, synapses are supposed to be involved in the propagation of altered proteins, and the loss of synapses is suggested as being causative regarding CI $[18,33,37,38]$. The changes in the synapses are suggested as being induced by ADNC, but the exact process is yet to be discovered [18, 33, 37, 38].

Here, we selected only a few samples to assess the SAF/SYP. The selection was based on neuronal counts, two with the highest and two with the lowest SAF/ NeuN. These four cases were selected, disregarding the ADNC pathology or age. We noted a slight decrease in 
the SAF/SYP in patients with a higher neuronal density. This finding suggests that a depletion of the matrix might be an early change, preceding a neuronal loss, i.e., the processes are altered prior to the cell loss. This observation is certainly questionable due to the low number of subjects assessed, but it emphasizes that studies on matrix versus neuronal somata in degenerative diseases are certainly warranted.

Based on our and previous results, non-invasive-, in vivo-, techniques to assess ADNC in iNPH is certainly of interest. Currently, there is the Positron Emission Tomography (PET), technique applying specific tracers to assess the deposits of $\mathrm{A} \beta$ and HPt in the brain [19, 29]. The most widely used is Carbon-11 labelled thioflavin-T derivate, Pittsburgh compound B $\left[{ }^{11} \mathrm{C}\right] \mathrm{PIB}$, to visualize $A \beta$, considered as being a reliable marker of $\mathrm{A} \beta$ pathology even in biopsied patients with iNPH [19, $34,39]$. In contrast, a HPt selective tracer $\left[{ }^{18} \mathrm{~F}\right] \mathrm{THK}-$ 5117 did not reflect the HPt pathology in the cortical biopsies from iNPH subjects $[24,29]$. The latter observation is due to the extremely low load of HPt pathology as observed here.

The low SAF/HPt and the observation of high neuronal density in a setting of iNPH suggest that neuroprotective-, antiapoptotic-, and HPт targeting therapies may benefit these patients by delaying or slowing down the neurodegenerative process that eventually will lead to $\mathrm{AD}$, as previously reported [2, $12,20,25,41,43]$. Furthermore, there is an urgent need to learn more about the constitutions of the matrix to eventually identify new targets for therapy.

Two-thirds of all patients affected by AD are females; thus, the female gender, next to age and apolipoprotein E status, has been reported as being one of the risk factors of $\mathrm{AD}$ [4]. In line with the above, in our cohort of subjects with iNPH, a higher number of females displayed ADNC when compared with men. This was noted particularly for HPt (both incidence and extent) but also at a borderline level for $\mathrm{A} \beta$ (both incidence and extent). Thus, our observations detected in a minimal cortical biopsy are in line with previously published data from several PM studies, where the whole brain has been investigated $[15,30]$.

Most studies assessing ADNC and neuronal population are performed on PM brain tissue, animal models, or cell cultures. We have unique material to study ADNC and neuronal cell counts in surgical brain biopsies from patients with iNPH. Due to the relatively large number of cases that have been sampled during 10 years, we were able to select a relatively homogenous cohort regarding the age. The absolute benefit while assessing surgical samples in contrast to PM tissue is that alterations to be looked for in the tissue are not affected by factors caused by agonal state or PM events. Furthermore, as previously reported, prospective analysis of tissue alteration, in relation to the clinical progression can be carried out $[22,25]$.

ADNC pathology is associated with cognitive decline and neuronal damage as well as neuronal death, which are observed in $\mathrm{AD}[10,16,28,38]$. These alterations are not expected to be observed in subjects with iNPH. The observation of ADNC in a cortex biopsy is however in line with the observation that a substantial number of subjects with iNPH develop AD over time [20]. Twenty years of experience while assessing tiny brain biopsies obtained during VPS operation of patients with iNPH and clinical prospective studies of these patients have increased our understanding of both iNPH and as well of $\mathrm{AD}$ related neurodegeneration. Based on the above, iNPH seems to be an intriguing and reliable model of AD. The histopathological assessment of the brain tissue of iNPH subjects was carried out to assign a pathoanatomical diagnosis, i.e., with or without ADNC. It is thus surprising that this assessment is seldom performed in this setting.

In this study, we assessed the extent of protein expression in the tissue, applying a computerized morphometrical analysis. We chose to measure the SAF for all the proteins, independent of the compartment of expression to facilitate comparison. For the final SAF, pixels of adequate staining intensity were included. Thus, we feel that the methodology for morphometrical analysis is reliable.

The assessment of a surgical biopsy is certainly informative. However, a tiny biopsy represents only a fraction of a brain and does not represent all the neuroanatomical regions with various vulnerabilities displaying different pathologies. Thus, one needs to acknowledge the influence of sampling deficit on all the above.

In conclusion, here we assessed ADNC and neuronal markers in surgical brain biopsies from patients with iNPH. We identified remarkable neuronal preservation but also a substantial depletion of matrix/neuropil within our cohort. These findings are intriguing as they suggest that a loss of matrix/neuropil might be a hallmark lesion in iNPH. This is in line with the cardinal feature of iNPH, enlarged ventricles. Further, the loss of matrix parallel with preservation of neurons, might be an early sign of neurodegeneration. A substantial number of our patients displayed either $A \beta$ or HPt or both pathologies, even if the extent of HPt, was low. These observations are indicative of a progressive neurodegenerative process, result in line with the finding that a substantial number of iNPH patients develop dementia. Additionally, females displayed more advanced ADNC and especially HPt density in their biopsies, in line with the interpretation that the female gender is a risk factor for ADNC and AD. Surprisingly, we noted a positive correlation between neuronal count and $A \beta$, but only in 
females. These findings indicate that even subjects with $\mathrm{A} \beta$ in cortex might benefit of neuroprotective therapy. Two decades of experience while assessing brain biopsies from subjects with iNPH, certainly suggest that studying this patient category is a reliable model of AD. Our findings further suggest that a histological assessment of a brain biopsy obtained during a VPS procedure should always be carried out as a diagnostic procedure as it reveals information that cannot be obtained otherwise; hence, the obtained information can lead to a more tailored treatment strategy.

\section{Acknowledgements}

We acknowledge the work of Svetlana Popova for her skillful technical assistance, the Statisticon for their support regarding the choice of statistical methods to be used, and Meena Strömqvist for her critical reading of the manuscript.

\section{Authors' contributions}

Conceptualization and Methodology, S.L., and I.A; Investigation, S.L., Writing S.L., and I.A.; Funding Acquisition and Resources, I.A., Both authors read and approved the final manuscript.

\section{Funding}

The sources of financial support include local grants (ALF) from the Uppsala University Hospital and grants from the Hans Gabriel and Alice TrolleWachtmeister foundation in Sweden. There is no conflict of interest.

\section{Competing interests}

The authors declare that they have no competing interests.

Received: 20 May 2019 Accepted: 23 May 2019

Published online: 29 May 2019

\section{References}

1. Abu Hamdeh S, Virhammar J, Sehlin D, Alafuzoff I, Cesarini KG, Marklund N (2018) Brain tissue $A \beta 42$ levels are linked to shunt response in idiopathic normal pressure hydrocephalus. J Neurosurg 1:1-9. https://doi.org/10.3171/ 2017.7.JNS171005

2. Alafuzoff I, Helisalmi S, Heinonen EH, Reinikainen $\mathrm{K}$, Hallikainen M, Soininen $\mathrm{H}$ et al (2000) Selegiline treatment and the extent of degenerative changes in brain tissue of patients with Alzheimer's disease. Eur J Clin Pharmacol 55: 815-819

3. Alafuzoff I, Thal DR, Arzberger T, Bogdanovic N, Al-Sarraj S, Bodi I et al (2009) Assessment of beta-amyloid deposits in human brain: a study of the BrainNet Europe Consortium. Acta Neuropathol 117:309-320. https://doi. org/10.1007/s00401-009-0485-4

4. Alzheimer's Association (2016) 2016 Alzheimer's disease facts and figures. Alzheimers Dement 12:459-509

5. Bech-Azeddine R, Høgh P, Juhler M, Gjerris F, Waldemar G (2007) Idiopathic normal-pressure hydrocephalus: clinical comorbidity correlated with cerebral biopsy findings and outcome of cerebrospinal fluid shunting. J Neurol Neurosurg Psychiatry 78:157-161

6. Braak H, Alafuzoff I, Arzberger T, Kretzschmar H, Del Tredici K (2006) Staging of Alzheimer disease-associated neurofibrillary pathology using paraffin sections and immunocytochemistry. Acta Neuropathol 112:389-404

7. Braak H, Braak E (1991) Neuropathological stageing of Alzheimer-related changes. Acta Neuropathol 82:239-259

8. Braak H, Del Tredici K (2011) The pathological process underlying Alzheimer's disease in individuals under thirty. Acta Neuropathol 121:171181. https://doi.org/10.1007/s00401-010-0789-4

9. Braak H, Thal DR, Ghebremedhin E, Del Tredici K (2011) Stages of the pathologic process in Alzheimer disease: age categories from 1 to 100 years. J Neuropathol Exp Neurol 70:960-969. https://doi.org/10.1097/NEN. Ob013e318232a379

10. Bussière T, Gold G, Kövari E, Giannakopoulos P, Bouras C, Perl DP et al (2003) Stereologic analysis of neurofibrillary tangle formation in prefrontal cortex area 9 in aging and Alzheimer's disease. Neuroscience 117:577-592
11. Cabral D, Beach TG, Vedders L, Sue LI, Jacobson S, Myers K et al (2011) Frequency of Alzheimer's disease pathology at autopsy in patients with clinical normal pressure hydrocephalus. Alzheimers Dement 7:509-513. https://doi.org/10.1016/j.jalz.2010.12.008

12. Congdon EE, Sigurdsson EM (2018) Tau-targeting therapies for Alzheimer disease. Nat Rev Neurol 14:399-415. https://doi.org/10.1038/s41582-0180013-z

13. Elobeid A, Laurell K, Cesarini KG, Alafuzoff I (2015) Correlations between mini-mental state examination score, cerebrospinal fluid biomarkers, and pathology observed in brain biopsies of patients with normal-pressure hydrocephalus. J Neuropathol Exp Neurol 74:470-479. https://doi.org/10. 1097/NEN.0000000000000191

14. Elobeid A, Libard S, Leino M, Popova SN, Alafuzoff I (2016) Altered Proteins in the Aging Brain. J Neuropathol Exp Neurol 75:316-325. https://doi.org/10. 1093/jnen/nlw002

15. Filon JR, Intorcia AJ, Sue LI, Vazquez Arreola E, Wilson J, Davis KJ et al (2016) Gender differences in Alzheimer disease: brain atrophy, histopathology burden, and cognition. J Neuropathol Exp Neurol 75:748-754. https://doi. org/10.1093/jnen/nlw047

16. Gómez-Isla T, Price JL, McKeel DW Jr, Morris JC, Growdon JH, Hyman BT (1996) Profound loss of layer II entorhinal cortex neurons occurs in very mild Alzheimer's disease. J Neurosci 16:4491-4500

17. Hyman BT, Phelps CH, Beach TG, Bigio EH, Cairns NJ, Carrillo MC et al (2012) National Institute on Aging-Alzheimer's Association guidelines for the neuropathologic assessment of Alzheimer's disease. Alzheimers Dement 8: 1-13. https://doi.org/10.1016/j.jalz.2011.10.007

18. Ingelsson M, Fukumoto $\mathrm{H}$, Newell KL, Growdon JH, Hedley-Whyte ET, Frosch MP et al (2004) Early Abeta accumulation and progressive synaptic loss, gliosis, and tangle formation in AD brain. Neurology 62:925-931

19. Klunk WE, Engler $H$, Nordberg A, Wang Y, Blomqvist G, Holt DP et al (2004) Imaging brain amyloid in Alzheimer's disease with Pittsburgh Compound-B. Ann Neurol 55:306-319

20. Koivisto AM, Kurki MI, Alafuzoff I, Sutela A, Rummukainen J, Savolainen S et al (2016) High Risk of Dementia in Ventricular Enlargement with Normal Pressure Hydrocephalus Related Symptoms. J Alzheimers Dis 52:497-507. https://doi.org/10.3233/JAD-150909

21. Kovacs GG, Xie SX, Lee EB, Robinson JL, Caswell C, Irwin DJ et al (2017) Multisite Assessment of Aging-Related Tau Astrogliopathy (ARTAG). J Neuropathol Exp Neurol 76:605-619. https://doi.org/10.1093/jnen/nlx041

22. Leinonen $V$, Koivisto AM, Savolainen S, Rummukainen J, Sutela A, Vanninen $R$ et al (2012) Post-mortem findings in 10 patients with presumed normalpressure hydrocephalus and review of the literature. Neuropathol Appl Neurobiol 38:72-86. https://doi.org/10.1111/j.1365-2990.2011.01195.x

23. Leinonen $V$, Koivisto AM, Savolainen S, Rummukainen J, Tamminen JN, Tillgren T et al (2010) Amyloid and tau proteins in cortical brain biopsy and Alzheimer's disease. Ann Neurol 68:446-453. https://doi.org/10.1002/ana. 22100

24. Leinonen V, Rauramaa T, Johansson J, Bottelbergs A, Tesseur I, van der Ark P et al (2018) S-[18F]THK-5117-PET and [11C]PIB-PET Imaging in Idiopathic Normal Pressure Hydrocephalus in Relation to Confirmed Amyloid- $\beta$ Plaques and Tau in Brain Biopsies. J Alzheimers Dis 64:171-179. https://doi. org/10.3233/JAD-180071

25. Libard S, Laurell K, Cesarini KG, Alafuzoff I (2018) Progression of Alzheimer's Disease-Related Pathology and Cell Counts in a Patient with Idiopathic Normal Pressure Hydrocephalus. J Alzheimers Dis 61:1451-1462. https://doi. org/10.3233/JAD-170446

26. Masliah E, Mallory M, Hansen L, DeTeresa R, Alford M, Terry R (1994) Synaptic and neuritic alterations during the progression of Alzheimer's disease. Neurosci Lett 174:67-72

27. Montine TJ, Phelps CH, Beach TG, Bigio EH, Cairns NJ, Dickson DW et al (2012) National Institute on Aging-Alzheimer's Association guidelines for the neuropathologic assessment of Alzheimer's disease: a practical approach. Acta Neuropathol 123:1-11. https://doi.org/10.1007/s00401-011-0910-3

28. Nelson PT, Alafuzoff I, Bigio EH, Bouras C, Braak H, Cairns NJ (2012) Correlation of Alzheimer disease neuropathologic changes with cognitive status: a review of the literature. J Neuropathol Exp Neurol 71:362-381. https://doi.org/10.1097/NEN.0b013e31825018f7

29. Okamura N, Furumoto S, Harada R, Tago T, Yoshikawa T, Fodero-Tavoletti M et al (2013) Novel 18F-labeled arylquinoline derivatives for noninvasive imaging of tau pathology in Alzheimer disease. J Nucl Med 54:1420-1427. https://doi.org/10.2967/jnumed.112.117341 
30. Oveisgharan S, Arvanitakis Z, Yu L, Farfel J, Schneider JA, Bennett DA (2018) Sex differences in Alzheimer's disease and common neuropathologies of aging. Acta Neuropathol 136:887-900. https://doi.org/10.1007/s00401-0181920-1

31. Pomeraniec IJ, Bond AE, Lopes MB, Jane JA Sr (2016) Concurrent Alzheimer's pathology in patients with clinical normal pressure hydrocephalus: correlation of high-volume lumbar puncture results, cortical brain biopsies, and outcomes. J Neurosurg 124:382-388. https://doi.org/10. 3171/2015.2.JNS142318

32. Power MC, Mormino E, Soldan A, James BD, Yu L, Armstrong NM et al (2018) Combined neuropathological pathways account for age-related risk of dementia. Ann Neurol 84:10-22. https://doi.org/10.1002/ana.25246

33. Rajmohan R, Reddy PH (2017) Amyloid-Beta and Phosphorylated Tau Accumulations Cause Abnormalities at Synapses of Alzheimer's disease Neurons. J Alzheimers Dis 57:975-999. https://doi.org/10.3233/JAD-160612

34. Rinne JO, Suotunen T, Rummukainen J, Herukka SK, Nerg O, Koivisto AM et al (2019) [11C]PIB PET Is Associated with the Brain Biopsy Amyloid- $\beta$ Load in Subjects Examined for Normal Pressure Hydrocephalus. J Alzheimers Dis 67: 1343-1351. https://doi.org/10.3233/JAD-180645

35. Savolainen S, Paljärvi L, Vapalahti M (1999) Prevalence of Alzheimer's disease in patients investigated for presumed normal pressure hydrocephalus: a clinical and neuropathological study. Acta Neurochir 141:849-853

36. Sethi MK, Zaia J (2017) Extracellular matrix proteomics in schizophrenia and Alzheimer's disease. Anal Bioanal Chem 409:379-394. https://doi.org/10. 1007/s00216-016-9900-6

37. Spires-Jones TL, Attems J, Thal DR (2017) Interactions of pathologica proteins in neurodegenerative diseases. Acta Neuropathol 134:187-205. https://doi.org/10.1007/s00401-017-1709-7

38. Spires-Jones TL, Hyman BT (2014) The intersection of amyloid beta and tau at synapses in Alzheimer's disease. Neuron 82:756-771. https://doi.org/10. 1016/j.neuron.2014.05.004

39. Thal DR, Beach TG, Zanette M, Lilja J, Heurling K, Chakrabarty A et al (2018) Estimation of amyloid distribution by $\left[{ }^{18} \mathrm{~F}\right]$ flutemetamol PET predicts the neuropathological phase of amyloid $\beta$-protein deposition. Acta Neuropatho 136:557-567. https://doi.org/10.1007/s00401-018-1897-9

40. Thal DR, Rüb U, Orantes M, Braak H (2002) Phases of A beta-deposition in the human brain and its relevance for the development of AD. Neurology 58:1791-1800

41. Wiciński M, Wódkiewicz E, Słupski M, Walczak M, Socha M, Malinowski B et al $(2018,2018)$ Neuroprotective Activity of Sitagliptin via Reduction of Neuroinflammation beyond the Incretin Effect: Focus on Alzheimer's Disease. Biomed Res Int:6091014. https://doi.org/10.1155/2018/6091014

42. Williams MA, Malm J (2016) Diagnosis and Treatment of Idiopathic Normal Pressure Hydrocephalus. Continuum (Minneap Minn) 22:579-599. https:// doi.org/10.1212/CON.0000000000000305

43. Youdim MBH (2018) Monoamine oxidase inhibitors, and iron chelators in depressive illness and neurodegenerative diseases. J Neural Transm 125: 1719-1733. https://doi.org/10.1007/s00702-018-1942-9

\section{Publisher's Note}

Springer Nature remains neutral with regard to jurisdictional claims in published maps and institutional affiliations.

Ready to submit your research? Choose BMC and benefit from:

- fast, convenient online submission

- thorough peer review by experienced researchers in your field

- rapid publication on acceptance

- support for research data, including large and complex data types

- gold Open Access which fosters wider collaboration and increased citations

- maximum visibility for your research: over $100 \mathrm{M}$ website views per year

At $\mathrm{BMC}$, research is always in progress.

Learn more biomedcentral.com/submissions 